\title{
Carcinoma de plexo coroide: relato de caso e revisão de literatura
}

\author{
Catarina Couras Lins ${ }^{1}$, Rodrigo Antonio Rocha da Cruz Adry², Leonardo Miranda \\ de Avellar ${ }^{3}$, Julia Ribeiro Araújo ${ }^{1}$, Marcio Cesar de Mello Brandão ${ }^{3,4}$ \\ Hospital Geral Roberto Santos
}

\section{RESUMO}

Tumores do plexo coroide são tumores raros de origem neuroectodérmica, Correspondem a $0,4 \%$ a $0,6 \%$ de todos os tumores cerebrais, com incidência anual de 0,3 caso por milhão. Em sua maioria, são tumores benignos, raramente se apresentando na forma maligna. Os locais mais comumente relatados são os ventrículos. Relato de caso: Trata-se de carcinoma de plexo coroide em criança de 6 anos de idade que iniciou quadro com cefaleia, paresia do oculomotor, evoluindo com distúrbio de marcha. Foi submetida à biopsia, com confirmação histopatológica do carcinoma de plexo coroide. Esse paciente foi submetido à cirurgia, evolui bem com recuperação quase total no pós-cirúrgico imediato.

\section{PALAVRAS-CHAVE}

Tumor, carcinoma, plexo coroide, neoplasia.

\begin{abstract}
Choroid plexus carcinoma: case report and literature review

Tumors of the choroid plexus are rare tumors of neuro-ectodermal origin, correspond to $0.4 \%$ to $0.6 \%$ of all brain tumors, with an annual incidence of 0.3 cases per million. They are mostly benign tumors, rarely presenting as malignant. The sites most frequently reported are the ventricles. Case report: This is choroid plexus carcinoma in ma child of 6 years of age she developed headache, oculomotor paresis progressing to gait disturbance. The patient underwent biopsy with histopathologic confirmation of choroid plexus carcinoma. This patient was submitted to surgery and progressed well, with full recovery in the immediate postoperative.
\end{abstract}

\section{KEYWORDS}

Tumor, carcinoma, choroid plexus, neoplasia.

\section{Introdução}

Tumores do plexo coroide são tumores raros de origem neuroectodérmica. ${ }^{11}$ Correspondem a $0,4 \%$ a $0,6 \%$ de todos os tumores cerebrais, ${ }^{19}$ com incidência anual de 0,3 caso por milhão. ${ }^{6,7}$ Em sua maioria, são tumores benignos, raramente se apresentando na forma maligna. ${ }^{9}$ Os locais mais comumente relatados são os ventrículos. ${ }^{21}$ No entanto, tumores extraventriculares foram descritos. ${ }^{13}$ Os tumores podem estar presentes em adultos e no período pré-natal, no entanto a maioria dos casos ocorre em crianças menores de 2 anos de idade. ${ }^{18}$ Os critérios histológicos para o diagnóstico desses tumores foram descritos por Lewis (1967) e, mais tarde, revisados por Kleihues e Cavenee (1997), incluindo invasão do tecido neural adjacente, perda da arquitetura papilar com achados de malignidade celular e variações marcantes do tamanho nuclear e da cromatina. ${ }^{8}$ No entanto, essa classificação histológica não está bem clara. ${ }^{9} \mathrm{O}$ tratamento dessas crianças é muitas vezes difícil e complicado por dilemas associados como a escolha dos procedimentos cirúrgicos, o tratamento da hidrocefalia, o controle de vascularização do tumor e o valor da terapia adjuvante. O presente estudo descreve um caso clínico de um paciente com carcinoma do plexo coroide.

\section{Relato do caso}

FVCO, sexo masculino, 6 anos de idade, apresentando há três meses cefaleia de leve intensidade em

1. Acadêmica de Medicina da Escola Bahiana de Medicina e Saúde Pública (Bahiana).

2. Médico-Residente em Neurocirurgia do Hospital de Base de São José do Rio Preto da Faculdade de Medicina de São José do Rio Preto (Famerp), Médico do Serviço de Neurologia e Neurocirurgia do Hospital Geral do Estado da Bahia e Hospital Geral Roberto Santos.

3. Preceptor da Residência Médica em Neurocirurgia do Hospital Geral Roberto Santos.

4. Chefe do Serviço de Neurologia e Neurocirurgia do Hospital Geral do Estado da Bahia e Hospital Geral Roberto Santos. 
região frontal, associada a edema palpebral à direita, esclerótica direita avermelhada e desvio lateral do globo ocular direito, evoluindo, 15 dias após o início dos sintomas, com vômitos frequentes, vertigem, ptose palpebral à direita, sonolência excessiva e dificuldade para deambular. Ao exame físico apresentava paresia do III par, diplopia, reflexo fotomotor direto ausente à direita, paraparesia crural, com força grau III em membro superior esquerdo e grau II em membro inferior esquerdo, hiper-reflexia em hemicorpo esquerdo com Babinski presente. A ressonância magnética evidenciou lesão expansiva intraventricular, irregular, isointensa ao córtex cerebral em T1 e T2, de textura relativamente heterogênea, discretamente hiperintensa em FLAIR, sugerindo caráter hidratado com discreta orla de hipersinal na substância branca periventricular, especialmente nos fórceps menores (pontas anteriores dos ventrículos laterais), por passagem de liquor sob pressão através do epêndima (transudação liquórica transependimária). Com contraste, há forte impregnação (Figura 1). O paciente foi submetido à biópsia, com retirada de amostra para estudo anatomopatológico, na qual a microscopia demonstrou secções com proliferação celular neoplásica composta por células grandes irregulares, moderada a intensamente atípicas, ora bizarras, exibindo numerosas figuras de mitose típicas e atípicas, formando arranjos papilares, pseudoglandulares e sólidos; áreas de necrose e hemorragia; vasos grandes e dilatados com paredes espessadas e focos de calcificação, concluindo se tratar de carcinoma de plexo coroide grau III da OMS de 2007. O paciente foi submetido à ressecção do tumor e está em tratamento com radioterapia. No anatomopatológico, verificou-se neoplasia indiferenciada em meio a tecido de plexo coroide de padrão epitelial com áreas de necrose e sinais de grande atividade mitótica (Figura 2).

\section{Discussão}

Os tumores do plexo coroide são raros, representam menos de $1 \%$ de todos os tumores intracranianos e são mais prevalentes antes de 2 anos de idade..$^{18} \mathrm{~A}$ etiologia dos tumores do plexo coroide tem sido relacionada a infecções pelo Simian Virus 40 (SV40). ${ }^{10}$ Entretanto, ainda não se sabe a sua real importância na etiologia desses tumores. ${ }^{8}$ O surgimento desses tumores também pode ser influenciado por outros fatores como as síndromes ligadas ao cromossomo $\mathrm{X}^{8} \mathrm{O}$ diagnóstico definitivo de um tumor do plexo coroide só pode ser obtido por meio da avaliação histológica. ${ }^{21}$ No entanto, deve-se fazer diagnóstico diferencial com os papilomas do plexo coroide, ependimomas papilares, meningiomas, meduloblastomas, astrocitomas e adenoma pituitário. ${ }^{4}$ Os tumores do plexo

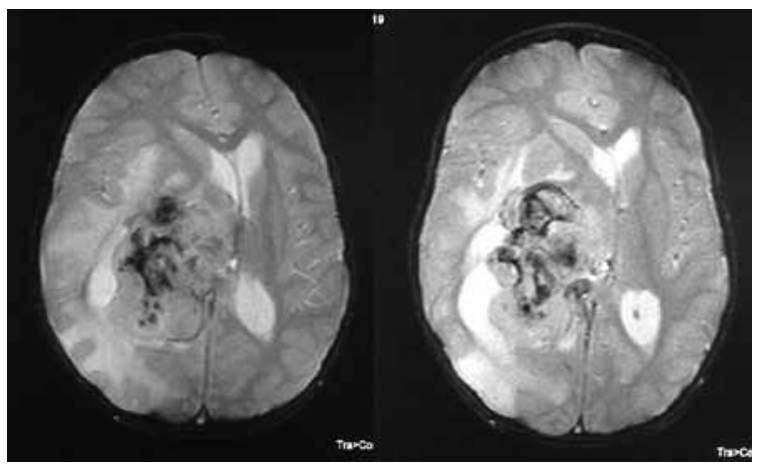

Figura 1 - Ressonância magnética mostrando carcinoma de plexo coroide em ventrículo lateral à direita.

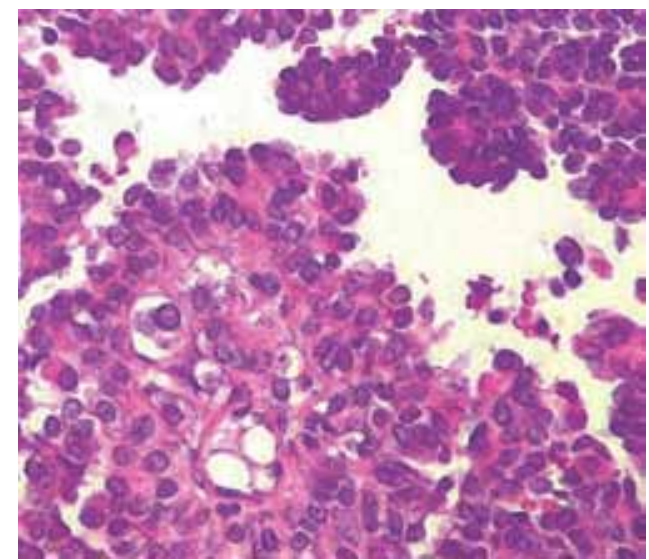

Figura 2 - Neoplasia indiferenciada em meio a tecido de plexo coroide de padrão epitelial com áreas de necrose e sinais de grande atividade mitótica.

coroide são classificados como carcinomas do plexo coroide (CPC, grau III, OMS $)^{8}$ e mostram epitélio colunar pseudoestratificado, com sinais francos de malignidade: acentuado pleomorfismo nuclear, mitoses frequentes, relação núcleo-citoplasma alta, densidade celular aumentada, necrose focal, proliferação vascular e perda da delimitação entre estroma e parênquima. ${ }^{15}$ Além disso, esses tumores frequentemente expressam antígeno carcinoembrionário $(\mathrm{CEA})^{15}$ e CD $44{ }^{20}$ que também pode ser detectado como marcador no sangue periférico. ${ }^{5} \mathrm{~A}$ negatividade para o CEA é útil na diferenciação com os adenocarcinomas metastáticos, embora alguns estudos relatem positividade em até $50 \%$ dos casos dos CPC2,9. Em diferentes estudos, a positividade para CK varia de $83 \%-100 \%$, para a S- 100 , de $40 \%-94 \%$, para VIM, de $16 \%-88 \%$, para a EMA, de 69\%-71\%, para a GFAP, de $22 \%-85 \%$ e para a ENE, de 20\%-100\%2,8. ${ }^{1}$ Os antígenos HEA125 e BerEP4 também são utilizados nessa diferenciação, pois são positivos em mais de 95\% dos carcinomas metastáticos e somente em $10 \%$ dos CPC. Entretanto, a aquisição desses marcadores em nosso meio é dificultada. ${ }^{22}$ Enquanto os carcinomas do plexo coroide (CPC) são relatados como tendo prognóstico extremamente pobre, ${ }^{2}$ os papilomas do plexo coroide (CPP) são geralmente considerados como tumo- 
res benignos com resultados muito favoráveis em longo prazo. ${ }^{16} \mathrm{~A}$ localização mais comum em crianças é o átrio ou o trígono dos ventrículos laterais (85\%). ${ }^{4} \mathrm{~A}$ incidência é maior no gênero masculino $(1: 1,3){ }^{4}$ As manifestações clínicas dos CPC variam com a localização do tumor. Quando em ventrículos laterais e terceiro ventrículo, geram hidrocefalia nos dois a três meses que antecedem o diagnóstico em crianças de baixa idade. Quando acometem o quarto ventrículo, sinais cerebelares podem associar-se ao quadro. As características radiológicas dos tumores do plexo coroide são inespecíficas. A tomografia axial computadorizada mostra tumor intraventricular heterogêneo com invasão do parênquima e graus variáveis de hidrocefalia. A ressonância magnética oferece melhor visualização das relações anatômicas do tumor e invasão do parênquima, facilitando a cirurgia e a avaliação pós-operatória. A administração conjunta de contrastes permite maior especificidade e sensibilidade na avaliação de tumores recorrentes e residuais. ${ }^{3}$ Em crianças de baixa idade, pode ser realizada ultrassonografia transfontanela, que mostrará massa intraventricular ecogênica invadindo o parênquima cerebral. ${ }^{8}$ Apesar do advento das técnicas de diagnóstico no pré-operatório e da moderna intervenção operacional terem reduzido dramaticamente a morbidade cirúrgica e as taxas de mortalidade, ${ }^{12} \mathrm{o}$ cirurgião ainda enfrenta uma série de problemas e dilemas quanto à escolha do procedimento cirúrgico, da vascularização do tumor, do tratamento da hidrocefalia e do valor da terapia adjuvante. Em geral, acredita-se que a ressecção cirúrgica é um importante elemento no tratamento. A ressecção do tumor é um poderoso passo terapêutico. A importância positiva da cirurgia parece ser maior do que na maioria dos outros tumores cerebrais. Isso suporta uma segunda cirurgia quando a radioimagem pós-cirúrgica mostra tumor residual. No pós-operatório, as crianças com carcinoma de plexo coroide são frequentemente tratadas com quimioterapia adjuvante. ${ }^{3} \mathrm{O}$ comportamento biológico do CPC é desfavorável, apesar do tratamento, com tempo médio de sobrevida em adultos, de 3,5 anos, e em crianças, de nove meses. ${ }^{16}$

\section{Conclusão}

O carcinoma de plexo coroide é um tumor pouco frequente, que geralmente não provoca sintomas em sua fase inicial, mas apenas quando apresenta efeito de massa importante ou fechamento do circuito do líquido cefalorraquidiano. O tratamento deve ser realizado por equipe multidisciplinar, utilizando-se diversos métodos, além da cirurgia, para uma resolução satisfatória.

\section{Referências}

1. Ang LC, Taylor AR, Bergin D, Kaufmann JCE. An immunohistichemical study of papillary tumors in the central nervous system. Cancer. 1990;15:2712-9.

2. Berger C, Thiesse P, Lellouch TA, Kalifa C, Pierre KA, Bouffet E. Choroid plexus carcinomas in childhood: clinical features and prognostic factors. Neurosurgery. 1998;42:470-5.

3. Bleggi-Torres LF, Noronha L, Telles JE. A importância da imunoistoquímica no diagnóstico anatomopatológico em hospital geral: análise de 885 casos. J Bras Patol. 1995;31:65-71.

4. Cila A, Ozturk C, Senaati S. Bilateral choroid plexus carcinoma of the lateral ventricles. Pediatr Radiol. 1992;22:136-7.

5. Hashizume A, Kodama Y, Hotta T, Yuki K, Taniguchi E, Eguchi $\mathrm{K}$, et al. Choroid plexuscarcinoma in the lateral ventricle case report. Neurol Med Chir (Tokyo). 1995;35:742 -4.

6. Janisch W, Staneczek W. Epidemiology of tumors of the central nervous system: influence of the autopsy rate on the incidence rate. Arch Geschwulstforsch. 1988;58:51-5.

7. Janisch W, Staneczek W. Primary tumors of the choroid plexus Frequency, localization and age. Zentralbl Allg Pathol. 1989;135:235-40.

8. Kleihues P, Cavenee WK. Tumours of the nervous system. Lyon: International Agency for Research on Cancer; 1997. p. 57-60.

9. Bleggi-Torres LF, Urban LABD, Antoniuk A, Carboni P, Ramina R, Gugelmin ES. Carcinoma de plexo coroide: relato de quinze casos. Arq Neuropsiquiatr. 2000;58(2-B):505-11.

10. Martini F, laccheri L, Lazzarin L, Carinci P, Corallini A, Gerosa $M$, et al. SV40 early region and large $T$ antigen in human brain tumor, peripheral blood cell, and sperm fluids from healthy individuals. Cancer Res. 1996;56:4820-5.

11. McEvoya AW, Hardinga BN, Phippsa PK, Ellisonb DW, Elsmorea AJ, Thompsona D, et al. Management of choroid plexus tumours in children: 20 years experience at a single neurosurgical centre. Pediatr Neurosurg. 2000;32:192-9.

12. McGirr SJ, Ebersold MJ, Scheithauer BW, Quast LM, Shaw EG. Choroid plexus papillomas: Long-term follow-up results in a surgically treated series. J Neurosurg. 1988;69:843-9.

13. Nakano I, Kondo A, Iwasaki K. Choroid plexus papilloma in the posterior third ventricle: case report. Neurosurgery. 1997;40:1279-82.

14. Packer RJ, Perilongo G, Johnson DC, et al. Choroid plexus carcinomas of childhood. Cancer. 1992;69:580-5.

15. Paulus W, Janisch W. Clinicopathologic correlations in epithelial choroid plexus neoplasm: a study of 52 cases. Acta Neuropathol. 1990;80:635-41.

16. Pencalet P, Sainte RC, Lellouch TA, Kalifa C, Brunelle F, Sgouros S, et al. Papillomas and carcinomas of the choroid plexus in children. J Neurosurg. 1998;88:521-8.

17. Pierga JY, Kalifa C, Terrier-Lacombe MJ, Habrand JL, Lemerle J. Carcinoma of the choroid plexus: a pediatric experience. Med Ped Oncol. 1993;21:480-7.

18. Sarkar C, Sharma MC, Gaikwad S, Sharma C, Singh VP. Choroid plexus papilloma: a clinicopathological study of 23 cases. Surg Neurol. 1999;52:37-9.

19. Tagle MP, Barriga CF, Huete LI. Carcinoma de plexos coroídes: utilidad de un tratamiento combinado. Rev Chil Pediatrc. 1994;65:276-80.

20. Varga Z, Vajtai I, Marino S, Schauble B, Yonekawa Y, Aguzzi. Tubular adenoma of the choroid plexus: evidence for glandular differentiation of the neuroepithelium. Pathol Res Pract. 1996;192:840-4.

21. Wolff JEA, Sajedi M, Brant R, Coppes MJ, Egeler RM. Choroid plexus tumours. Br J Cancer. 2002;87:1086-109.

22. Yuasa H, Tokito S, Tokunaga M. Primary carcinoma of the choroid plexus in Li-Fraumeni syndrome: case report. Neurosurgery. 1993;32:131-4.

Endereço para correspondência

Catarina Couras

Av. Sete de Setembro, 690, ap. 704, Bairro Dois de Julho

40060-001 - Salvador, BA

Telefone: (71) 8883-3665

E-mail: catarinacouras@hotmail.com 